\begin{tabular}{|c|c|}
\hline Title & Rapid immunohistochemistry based on al ternating current electric field for intraoperative diagnosis of brain tumors \\
\hline Author(s) & $\begin{array}{l}\text { T anino, Mishie; Sasaj ima, T oshio; Nanjo, Hiroshi; A kesaka, Shiori; Kagaya, Masami; Kimura, Taichi; Ishida, Y usuke; } \\
\text { Oda, Masaya; Takahashi, Masataka; Sugawara, T aku; Y oshioka, T oshiaki; Nishihara, Hiroshi; A kagami, Y oichi; Goto, } \\
\text { A kiteru; Minamiya, Y oshihiro; T anaka, Shinya }\end{array}$ \\
\hline Citation & $\begin{array}{l}\text { Brain tumor pathology, 32(1), 12-19 } \\
\text { https://doi.org/10.1007/s10014-014-0188-y }\end{array}$ \\
\hline Issue Date & 2015-01 \\
\hline Doc URL & http:/hdl.handle.net/2115/60447 \\
\hline Rights & The final publication is available at link.springer.com \\
\hline Type & article (author version) \\
\hline File Information & manuscript.pdf \\
\hline
\end{tabular}

Instructions for use 


\section{Rapid immunohistochemistry based on alternating current electric field for intraoperative diagnosis of brain tumors}

Mishie Tanino* ${ }^{1}$, Toshio Sasajima*2, Hiroshi Nanjo ${ }^{3}$, Shiori Akesaka ${ }^{1}$, Masami Kagaya ${ }^{5}$, Taichi Kimura ${ }^{1}$, Yusuke Ishida ${ }^{1}$, Masaya Oda $^{2}$, Masataka Takahashi ${ }^{2}$, Taku Sugawara ${ }^{2}$, Toshiaki Yoshioka ${ }^{3}$, Hiroshi Nishihara ${ }^{6}$, Yoichi Akagami ${ }^{5}$, Akiteru Goto ${ }^{3}$, Yoshihiro Minamiya $^{4}$, Shinya Tanaka, ${ }^{1,6}$, R-IHC Study Group ${ }^{7}$.

*: These authors equally contributed to this work.

${ }^{1}$ Department of Cancer Pathology, Hokkaido University Graduate School of Medicine, N15W7, Kita-ku, Sapporo 060-8638, ${ }^{2}$ Department of Neurosurgery, ${ }^{3}$ Division of Clinical Pathology, ${ }^{4}$ Department of Thoracic Surgery, Akita University Graduate School of Medicine, 1-1-1 Hondo, Akita 010-8543, ${ }^{5}$ Akita Industry Technology Center, Sanuki, Aza, Arayamachi, Akita 010-1623, ${ }^{6}$ Department of Translational Pathology, Hokkaido University Graduate School of Medicine, N15W7, Kita-ku, Sapporo 060-8638, Japan.

Corresponding author

Shinya Tanaka, M.D., Ph.D.

Department of Cancer Pathology, Hokkaido University Graduate School of Medicine N15, W7, Kita-ku, Sapporo 060-8638, Japan.

e-mail: tanaka@med.hokudai.ac.jp

TEL +81(11)-706-5052, FAX +81(11)-706-5902 


\section{Abstract}

Rapid immunohistochemistry (R-IHC) can contribute to the intraoperative diagnosis of central nervous system (CNS) tumors. We have recently developed a new IHC method based on an alternating current (AC) electric field to facilitate the antigen-antibody reaction. To ensure the requirement of R-IHC for intraoperative diagnosis, 183 cases of CNS tumors were reviewed regarding the accuracy rate of diagnosis without R-IHC. The diagnostic accuracy was $91.8 \%$ (168/183 cases) in which definitive diagnoses were not provided in 17 cases because of the failure of glioma grading and differential diagnosis of lymphoma and glioma. To establish the clinicopathological application, R-IHC for frozen specimens was compared with standard IHC for permanent specimens. 33 gliomas were analyzed, and the Ki-67/MIB-1 indices of frozen specimens by R-IHC were consistent with the grade and statistically correlated with those of permanent specimens. Thus, R-IHC provided supportive information to determine the grade of glioma. For discrimination between glioma and lymphoma, R-IHC was able to provide clear results of CD20 and Ki-67/MIB-1 in four frozen specimens of CNS lymphoma as well as standard IHC. We conclude that the R-IHC for frozen specimens can provide important information for intraoperative diagnosis of CNS tumors. (192 words)

Key words: Rapid immunohistochemistry (R-IHC), glioma, Central nervous system-lymhoma (CNS-lymphoma) 


\section{Introduction}

The importance of intraoperative pathological diagnosis has increased in recent years

[1]. Recently, in addition to the usefulness of CT and MRI images, additional multi-modal imaging techniques such as PET have improved the diagnostic accuracy. However, there are still limitations in obtaining an exact preoperative diagnosis [2] [3].

Pathological examination is performed based on the morphological findings such as cytological atypism, mitosis, vascular proliferation, and necrosis [4], in conjunction with the patient's clinical history, neuroradiologic images, and surgical findings. To date, the probability of intraoperative diagnosis has been reported as 66 to $95.6 \%$ [5] [6].

In the last three decades, several methods for Rapid immunohistochemistry (R-IHC) have been proposed that use microwave [7], high quality reagents [8] [9] [10] [11] [12], intermittent microwave [13], and ultrasound [14]. In these studies, only a few papers were focused on the application of these techniques for central nervous system (CNS) tumors [10] [11]. Recently, we have developed a novel R-IHC method based on alternating current (AC) electric field which facilitates the antigen-antibody reaction, and reported its usefulness for detection of sentinel lymph nodes metastasis of lung cancer [15].

In this study, to examine the diagnostic advantage of our newly developed R-IHC methods for rapid diagnosis of CNS tumor, we compared the results of R-IHC on frozen specimens with that of ordinal IHC on permanent specimens of CNS tumor, and evaluated their diagnostic accuracy by combination of H\&E staining and R-IHC in frozen sections. 
Materials and methods

CNS tumor specimens and the criteria for diagnostic accuracy

To evaluate the intraoperative diagnostic accuracy of CNS tumors, we reviewed 183 cases of all CNS tumors that were diagnosed intraoperatively from January 2008 to May 2013 in Department of Cancer Pathology, Hokkaido University Graduate School of Medicine. The final diagnosis were as follows: $42.7 \%$ (79/183 cases) of high grade glioma (HGG), 13.1\% (24/183 cases) of low grade glioma (LGG), $18.6 \%$ (34/ 183 cases) of metastatic carcinoma, $11.5 \%$ (21/ 183 cases) of meningioma, $4.3 \%$ (8/ 183 cases) of CNS lymphoma, $3.3 \%$ (6/ 183 cases) of schwannoma, $1.1 \%$ ( 2/183 cases) of craniopharyngioma, and $4.9 \%$ ( $9 / 183$ cases) of non-neoplastic lesions. We classified our diagnoses into the following three degrees correlated to the accuracy of intraoperative diagnosis according to the modified criteria shown in [16]: (1) The intraoperative diagnosis was the same as the final diagnosis which means correct tumor lineage and grade; low or high (complete correlation); (2) the intraoperative diagnosis was not incorrect but was too broad to qualify as a complete correlation (partial correlation); and (3) the intraoperative diagnosis was incorrect and different from the final diagnosis (no correlation)

\section{CNS tumor specimens for R-IHC}

We have performed R-IHC using 15 cases of glioma and four cases of CNS lymphoma from the specimens for intraoperative diagnosis at Hokkaido University that were unintentionally selected. Case 3 to 15 in glioma and case 37 in CNS lymphoma were 
performed R-IHC at the time of intraoperative diagnosis and Case 1, 2, 34, 35 and 36 were performed R-IHC in their recut frozen section that were kept in deep freezer after intraoperative diagnosis (Table 1a). 18 cases (Case 16 to 33) of glioma at the Department of Neurosurgery, Akita University Hospital were consecutively used for this study from September 2011 through May 2013 to compare the institutional differences (Table 1b). This study was approved by the Medical Ethics Committee of Hokkaido University Graduate School of Medicine and Akita University Hospital.

\section{Preparation of frozen and FFPE tissues}

Surgically resected specimens for intraoperative diagnosis were placed into the plastic cassette, and mounted with OCT compound medium (Sakura Finetek Japan Co., Ltd., Tokyo, Japan), then frozen by liquid nitrogen, referred to frozen sections were performed H\&E stain and R-IHC. Additionally resected tumors were fixed with $10 \%$ neutralized buffered formalin and embedded with paraffin. The Formalin-fixed paraffin-embedded (FFPE) tissues, referred to permanent sections were performed H\&E stain and ordinal IHC.

\section{R-IHC for frozen tissues}

R-IHC was performed using a newly developed machine as described[15]. Briefly, frozen tissues were sectioned at $5 \mu \mathrm{m}$ thicknesses, placed on slide glasses, and fixed by acetone at $4^{\circ} \mathrm{C}$ for $30 \mathrm{sec}$, and endogenous peroxidase (PO) was quenched by $3 \% \mathrm{H}_{2} \mathrm{O}_{2}$ at RT for $1 \mathrm{~min}$. Subsequently, the sections were incubated with primary antibody under a combination of 
high-voltage $(3.4 \mathrm{kV}$, offset $2.4 \mathrm{kV})$ and low-frequency $(18 \mathrm{~Hz})$ with altered current (AC) electric field for $5 \mathrm{~min}$. The sections were washed 3 times with PBS with $0.05 \%$ Tween 20 and incubated with EnVision TM+ System/HRP Mouse/ Rabbit (Dako, Glostrup, Denmark) for 5 min under a high-voltage $(3.4 \mathrm{kV}$, offset $2.4 \mathrm{kV})$ and low-frequency $(14 \mathrm{~Hz}) \mathrm{AC}$ electric field. Reagents that reacted with PO were visualized by diaminobenzidine (DAB) as a substrate at RT for 2 min., counterstained with hematoxylin, dehydrated, and mounted with coverslips. Except for the duration of the preparation of frozen tissue sections on the slide glass, approximately 16 min. were enough to accomplish whole process from PO quenching to obtain IHC slide glass for diagnosis. The machine was used during incubation period with primary or secondary antibodies to facilitate the reaction. R-IHC for Ki-67/MIB-1, CD3, and CD20 were performed using frozen sections that used for intraoperative diagnosis.

\section{Standard IHC for permanent tissues}

Standard IHC was performed as described elsewhere [15]. Briefly, sectioned specimens were incubated with primary antibody at RT for $60 \mathrm{~min}$. and washed with PBS with $0.05 \%$ Tween 20 for $5 \mathrm{~min} .3$ times, and then incubated with Envision at RT for $30 \mathrm{~min}$. Reacted antibodies were visualized by enzyme reaction with DAB as a substrate. Standard IHC for Ki-67/MIB-1, CD3, and CD20 were performed using FFPE sections that used for permanent diagnosis.

\section{Antibodies}


The following antibodies were used as the primary antibody with the appropriate dilution shown in parentheses: monoclonal mouse anti-Ki-67/MIB-1 antibody (monoclonal, clone MIB-1; Dako, 1:100), anti-CD20 antibody (monoclonal, clone L26; Dako, 1:400), and anti-CD3 antibody (polyclonal, rabbit; Dako, 1:200).

\section{Statistical analysis}

The correlation between frozen and permanent sections of Ki-67/MIB-1 indices were evaluated by Pearson's correlation coefficient. A value of $\mathrm{P}<0.05$ was considered as significant.

\section{Results}

\section{Limitation of the accuracy of intraoperative diagnosis of CNS tumors without R-IHC}

The overall diagnostic accuracy (the complete correlation) was 91.8\% (168/183 cases), and this was as high as the accuracy described in previous studies. The accuracy for each diagnosis was as follows; glioma 91\% (94/103), metastatic carcinoma 100\% (34/34), meningioma 90\% (19/21), CNS lymphoma $50 \%$ (4/8), shcwannoma 66\% (4/6), and craniopharyngioma $100 \%(2 / 2)$. The partial correlation was $8.2 \%(15 / 183$ cases $)$ and no correlation was $1.1 \%(2 / 183$ cases $)$. In these two no-correlation cases, we could not determine the glioma grading even as low or high grade, and failed to make a differential diagnosis of glioma from lymphoma. 
In case of the discrimination of low and high grade glioma, Ki-67/MIB-1 index may become supportive information, and R-IHC can be completed within $16 \mathrm{~min}$. and provide clear staining of Ki-67/MIB-1. Both in Case 6 and Case 13, H\&E staining for frozen specimens showed increased cell numbers with nuclear atypia suggesting diagnosis as grade II or III glioma (Fig. 1a and 1c). By using R-IHC, Ki-67/MIB-1 index in frozen specimens were $6.7 \%$ and $23 \%$, respectively (Fig. $1 \mathrm{~b}$ and $1 \mathrm{~d}$ ); thus, we considered Case 6 as being grade II and Case 13 as Grade III. These decisions were consistent with the final diagnosis using FFPE tissues with Ki-67/MIB-1 index in ordinal IHC (Fig. 1e, 1f, 1g, 1h and Table 1a). Application of intraoperative R-IHC for lymphocyte surface antigen for diagnosis of

\section{CNS lymphoma}

For the intraoperative diagnosis, discrimination between glioma and lymphoma is occasionally difficult without any support of R-IHC. Thus, we examined the application of lymphocyte surface antigen to R-IHC methods, and among the four cases of CNS lymphoma analyzed, CD20 and Ki-67/MIB-1 indices were successfully stained in all frozen samples by using R-IHC. Ki-67/MIB-1 indices were as high as those found in permanent sections (Fig. 2 and Table 2).

\section{Comparison of the results of Ki-67/MIB-1 in glioma between R-IHC for frozen tissues} and standard IHC for FFPE tissues

Clinicopathological application of the new R-IHC machine to CNS tumor diagnosis 
was evaluated. The diagnosis of H\&E stain with or without Ki-67/MIB-1 index in frozen sections and final diagnosis with immunohistochemstry in permanent sections of this study were shown in Table 1a and 1b. As measurement of Ki-67/MIB-1 index is critical for glioma grading, we compared the results of immunostaining of Ki-67/MIB-1 between R-IHC for frozen tissues and standard IHC for FFPE tissues in two independent facilities, Hokkaido University and Akita University.

Correlation of Ki-67/MIB-1 indices with glioma grading were observed with Grade II as $2.4 \pm 1.2(\mathrm{SD}) \%$, Grade III as $11.6 \pm 9.9(\mathrm{SD}) \%$, and Grade IV as $19.1 \pm 5.9(\mathrm{SD}) \%$ in Hokkaido University and Grade I as $0.5 \%$, Grade II as $0.8 \pm 1.1(\mathrm{SD}) \%$, Grade III as $13.0 \pm$ 8.2(SD)\%, and Grade IV as 29.6 \pm 16.3 (SD)\% in Akita University (Fig. 3a and 3b). In addition, the Ki-67/MIB-1 indices based on R-IHC for frozen sections significantly correlated with those of permanent sections in both Hokkaido University. $(\mathrm{P}<0.01, \mathrm{r}=0.87)$ and Akita University $(\mathrm{P}<0.01, \mathrm{r}=0.93)$ (Fig. $3 \mathrm{c}$ and $3 \mathrm{~d})$.

\section{Discussion}

We have established a new machine for R-IHC based on a novel principle of AC-facilitated antigen-antibody reaction, by which the turn-around time to obtain the results for surgeons in case of intraoperative diagnosis can be almost within $30 \mathrm{~min}$. It is obvious that this new R-IHC method can be applied for various specimens of the intraoperative diagnosis, but it should also possess advantages in the field of CNS tumor diagnosis. To 
ensure the requirement of R-IHC for the intraoperative diagnosis of CNS tumors, we have surveyed our 183 cases and found that the accuracy of the intraoperative diagnostic without IHC was $91.8 \%$, as same as in previous reports. Among them, the most difficult diagnosis was glioma grading, especially discrimination between grade II and grade III. In this study, we clarified that R-IHC for Ki-67/MIB-1 index can provide important information for glioma grading in two independent institutes. Ki-67/MIB-1 indices of R-IHC in frozen sections were statistically correlated to those of conventional IHC in permanent sections. In addition, discrimination of CNS lymphoma from glioma was occasionally difficult, and this new R-IHC method clearly demonstrated the positivity of the lymphocyte surface antigen CD20. Although R-IHC obviously provide further information about the tumor, the number of analyzed cases in this study was not enough to provide clear cut-off value for Ki-67/MIB-1 index according to the WHO grading. Furthermore, we should notice the limitation of intraoperative diagnosis using R-IHC mainly due to sampling divergency including tumor heterogeneiety in glioma, poor demarcation of CNS lymphoma, and differential location of sampling. Indeed, Ki-67/MIB-1 index by R-IHC in case 21 were not matched with that by standard IHC by the tumor heterogeneity.

Similar to our present study, several groups have reported the limitations of intraoperative diagnosis of CNS tumors such as glioma grading and determination of astrocytic versus oligodendroglial origin in addition to the differential diagnosis of CNS 
lymphoma, spindle cell lesions, reactive lesions as gliosis, poorly differentiated metastatic carcinoma, and primitive neuroectodermal tumor (PNET) [5] [11]. In this study, as we focused on the utility for diagnosis of glioma and CNS lymphoma by using limited number of antibodies as Ki-67/MIB-1, CD3 and CD20, we could not distinguish between astrocytic and oligodendroglial tumor. The combination of several markers will provide more precise diagnosis in the future. At least, to distinguish gliosis from low grade glioma, Ki-67/MIB-1 by R-IHC is useful as shown in case 4. In the near future, application of anti-IDH1-R132H antibody on this R-IHC method is expected to the discrimination of glioma and gliosis.

To date, several methods for R-IHC were proposed including microwave, specific reagent, and a combination of microwave and ultrasound, but such specific methods have not been widely accepted in the field of clinicopathological diagnosis for several reasons, such as the requirement of higher concentrated primary antibody or non-specific reaction due to the possible increase of temperature of the specimens by microwave. By our new method, fine staining can be obtained by using standard or sometimes lower concentrations of primary antibodies compared to standard IHC methods (data not shown). As compared to another R-IHC methods for Ki-67/MIB-1, our method would be more beneficial from the standpoint of antibody concentration (Table 3 ). In addition, we have measured the temperature of the specimens within AC stimulation and found a constant temperature (data not shown).

In conclusion, the new R- IHC method using AC field provides reliable results of IHC 
for CNS tumor diagnosis on frozen sections, and will contribute to an appropriate

intraoperative rapid diagnosis.

\section{Acknowledgement}

This work was supported in part by the Japan Society for the Promotion of Science (JSPS)

Grant-in-Aid for Scientific Research (KAKENHI Grant Number 24590406) to M.T. and (KAKENHI Grant Number 23390311) to Y.M.

R-IHC Study Group; Shinya Tanaka, Mishie Tanino, Tomoko Takenami, Shiori Akesaka, Manami Watanabe, Eiko Aoyanagi (Hokkaido University), Akira Kurose, Emiko Mizuki, Naoya Kumagai (Hirosaki Universiity), Yu Sugai, Noriyuki Yamada, Chikako Tomizawa (Iwate Medical University), Mareyuki Endo, Miki Aoki, Akira Morohashi, Tomoko Konta (Sendai Kousei Hospital), Kiyotaka Onodera, Manabu Suzuki, Yoshiki Kogi, Satoshi Ota, Yukio Nakatani (Chiba University), Takeo Yano, Tokuyoshi Maruyama, Tomohide Ogura, Jyunya Takeyama, Kazuki Kaneyama, Yoshiyuki Omura, Taizo Shiraishi (Mie University), Tomoo Ito, Yasuhiro Sakai, Emii Yanagida, Naoko Imagawa, Hiroshi Yamada, Tatsuko Tsukamoto (KobeUniversity), Shiro Takegami (Tohoku University), Satoru Kamata, Eichi Suzuki, Yoichi Akagami, Masami Kagaya, Ryuta Nakamura (Akita Industrial Technology Center), Shunsuke Wakayama, Yoshihiro Minamiya, Toshio Sasajima, Akiteru 
Goto, Hiroshi Nanjyo, Satoshi Ito, Hayato Konno, Yashushi Kawaharada, Shinnosuke Watanabe,

Tomoaki,Yoshioka, Kasumi Narita, Naoko Takahashi, Satoshi Kudou (Akita University) 


\section{Figure legends}

Fig. 1. Histological findings in Case 6. (a. H\&E staining in frozen specimen; b.

Ki-67/MIB-1 staining by R-IHC in frozen specimen; c. H\&E staining in FFPE specimen; d. Ki-67/MIB-1 staining by standard IHC in FFPE specimes). Histological findings in Case 13. (e. H\&E staining in frozen specimen; f. Ki-67/MIB-1 staining by R-IHC in frozen specimen; g. H\&E staining in FFPE specimen; h. Ki-67/MIB-1 staining by standard IHC in FFPE specimen). Scale bars, $20 \mu \mathrm{m}$.

Fig. 2. Histological findings of frozen specimen in Case 37 (a. H\&E staining; b. CD20 staining by R-IHC; c.CD3 staining by R-IHC; d. Ki-67/MIB-1 staining by R-IHC), and FFPE specimens for final diagnosis (e. H\&E staining; f. CD20 staining by standard IHC; g.CD3 staining by standard IHC ; h. Ki-67/MIB-1 staining by standard IHC). Scale bars, $20 \mu \mathrm{m}$.

Fig. 3. Ki-67/MIB-1 indices in frozen and FFPE specimens in glioma. a. Ki-67/MIB-1 indices by R-IHC in 15 frozen specimens of grade II to IV glioma at Hokkaido University. b. Ki-67/MIB-1 indices by R-IHC in 18 frozen specimens of grade I to IV glioma at Akita University Hospital. c. Correlation between Ki-67/MIB-1 indices by R-IHC using frozen specimens and those by standard IHC using FFPE specimens at Hokkaido University d. Correlation between Ki-67/MIB-1 indices by R-IHC using frozen specimens and those by standard IHC using FFPE specimens at Akita University Hospital. 


\section{References}

1. Brat DJ, Prayson RA, Ryken TC, et al. (2008) Diagnosis of malignant glioma: role of neuropathology. J Neurooncol.89(3):287-311.

2. Faehndrich J, Weidauer S, Pilatus U, et al. (2011) Neuroradiological viewpoint on the diagnostics of space-occupying brain lesions. Clin Neuroradiol.21(3):123-39.

3. Nihashi T, Dahabreh IJ, Terasawa T (2013) Diagnostic accuracy of PET for recurrent glioma diagnosis: a meta-analysis. AJNR Am J Neuroradiol.34(5):944-50, S1-11.

4. Louis DN, Ohgaki H, Wiestler OD, et al. (2007) WHO Classification of Tumors of the Central nervous system. IARC: Lyon.

5. Plesec TP, Prayson RA (2007) Frozen section discrepancy in the evaluation of central nervous system tumors. Arch Pathol Lab Med.131(10):1532-40.

6. Uematsu Y, Owai Y, Okita R, et al. (2007) The usefulness and problem of intraoperative rapid diagnosis in surgical neuropathology. Brain Tumor Pathol.24(2):47-52.

7. Ichihara T, Nakao A, Suzuki Y, et al. (1989) Improvement of the rapid immunoperoxidase staining method for intraoperative pathological diagnosis of pancreatic cancer using microwave irradiation. J Surg Oncol.42(3):209-14.

8. Richter T, Nahrig J, Komminoth P, et al. (1999) Protocol for ultrarapid immunostaining of frozen sections. J Clin Pathol.52(6):461-3.

9. Kammerer U, Kapp M, Gassel AM, et al. (2001) A new rapid immunohistochemical 
staining technique using the EnVision antibody complex. J Histochem

Cytochem.49(5):623-30.

10. Haapasalo J, Mennander A, Helen P, et al. (2005) Ultrarapid Ki-67 immunostaining in frozen section interpretation of gliomas. J Clin Pathol.58(3):263-8.

11. Uzuka T, Aoki H, Natsumeda M, et al. (2011) Indication of intraoperative immunohistochemistry for accurate pathological diagnosis of brain tumors. Brain Tumor Pathol.28(3):239-46.

12. Monig SP, Luebke T, Soheili A, et al. (2006) Rapid immunohistochemical detection of tumor cells in gastric carcinoma. Oncol Rep.16(5):1143-7.

13. Hatta H, Tsuneyama K, Kumada T, et al. (2006) Freshly prepared immune complexes with intermittent microwave irradiation result in rapid and high-quality immunostaining. Pathol Res Pract.202(6):439-45.

14. Hatta H, Tsuneyama K, Kondo T, et al. (2010) Development of an ultrasound-emitting device for performing rapid immunostaining procedures. J Histochem Cytochem.58(5):421-8.

15. Toda H, Minamiya Y, Kagaya M, et al. (2011) A novel immunohistochemical staining method allows ultrarapid detection of lymph node micrometastases while conserving antibody. Acta Histochem Cytochem.44(3):133-9.

16. Firlik KS, Martinez AJ, Lunsford LD (1999) Use of cytological preparations for the 
intraoperative diagnosis of stereotactically obtained brain biopsies: a 19-year experience and survey of neuropathologists. J Neurosurg.91(3):454-8. 
Table 1. Summary of intraoperative and final findings

a. Hokkaido University

\begin{tabular}{|c|c|c|c|c|c|c|}
\hline Case & Age/Sex & $\begin{array}{c}\text { Frozen } \\
\text { HE }\end{array}$ & $\begin{array}{c}\text { MIB-1 } \\
\text { (R-IHC) }\end{array}$ & $\begin{array}{c}\text { Frozen } \mathrm{HE} \\
+\mathrm{R}-\mathrm{IHC}\end{array}$ & $\begin{array}{l}\text { Permanent } \\
\text { MIB-1 }\end{array}$ & $\begin{array}{c}\text { Final } \\
\text { diagnosis }\end{array}$ \\
\hline $1^{*}$ & $48 / \mathrm{F}$ & II & 3.5 & II & 3 & II \\
\hline $2^{*}$ & $37 / \mathrm{M}$ & II & 2.7 & II & 4.5 & II \\
\hline 3 & $62 / \mathrm{M}$ & II & 3.2 & II & 4 & II \\
\hline 4 & $37 / F$ & II or gliosis & 2.1 & II & 2.6 & II \\
\hline 5 & $16 / M$ & II & 0.5 & II & 0.1 & II \\
\hline 6 & $37 / \mathrm{M}$ & II or III & 6.7 & II & 5 & II \\
\hline 7 & $63 / F$ & III & 5 & III & 6 & III \\
\hline 8 & $55 / F$ & IV & 15.1 & IV & 15 & IV \\
\hline 9 & $83 / \mathrm{M}$ & IV & 16.7 & IV & 30 & IV \\
\hline 10 & $60 / F$ & IV & 15.6 & IV & 39.7 & IV \\
\hline 11 & $59 / \mathrm{M}$ & III or IV & 20.4 & III or IV & 25 & IV \\
\hline 12 & $39 / \mathrm{M}$ & IV & 12 & IV & 25 & IV \\
\hline 13 & $30 / F$ & II or III & 23 & III & 15 & III \\
\hline 14 & $81 / F$ & IV & 28 & IV & 50 & IV \\
\hline 15 & $65 / \mathrm{M}$ & IV & 25.8 & IV & 50 & IV \\
\hline
\end{tabular}

b. Akita University

\begin{tabular}{|c|c|c|c|c|c|c|}
\hline Case & Age/Sex & $\begin{array}{c}\text { Frozen } \\
\mathrm{HE}\end{array}$ & $\begin{array}{c}\text { MIB-1 } \\
(\mathrm{R}-\mathrm{IHC})\end{array}$ & $\begin{array}{c}\text { Frozen } \mathrm{HE} \\
+\mathrm{R}-\mathrm{IHC}\end{array}$ & $\begin{array}{l}\text { ermanent } \\
\text { MIB-1 }\end{array}$ & $\begin{array}{c}\text { Final } \\
\text { diagnosis }\end{array}$ \\
\hline 16 & $4 / F$ & I & 0.5 & 1 & 2 & I \\
\hline 17 & $32 / F$ & II & 2 & I & 8 & II \\
\hline 18 & $43 / F$ & II & 0.2 & I & 1.8 & II \\
\hline 19 & 49/M & II or III & 0.1 & II & 2.7 & II \\
\hline 20 & $29 / M$ & IIor III & 15 & III & 17.9 & III \\
\hline 21 & $32 / F$ & II & 4 & II & 12.7 & III \\
\hline 22 & $33 / \mathrm{M}$ & II or III & 20 & III & 25.6 & III \\
\hline 23 & $46 / F$ & III or IV & 15 & III or IV & 23 & IV \\
\hline 24 & $50 / F$ & IV & 25 & IV & 36.7 & IV \\
\hline 25 & $60 / \mathrm{M}$ & IV & 30 & IV & 28.9 & IV \\
\hline 26 & $63 / \mathrm{M}$ & IV & 12 & IV & 14.3 & IV \\
\hline 27 & $65 / \mathrm{M}$ & IV & 39.5 & IV & 77.4 & IV \\
\hline 28 & $69 / F$ & IV & 26.7 & IV & 40.4 & IV \\
\hline 29 & $71 / F$ & IV & 25 & IV & 40 & IV \\
\hline 30 & $72 / \mathrm{M}$ & IV & 30 & IV & 26.7 & IV \\
\hline 31 & $74 / F$ & III or IV & 46 & IV & 81 & IV \\
\hline 32 & $76 / F$ & IV & 10 & IV & 11.7 & IV \\
\hline 33 & $87 / \mathrm{M}$ & IV & 66 & IV & 71 & IV \\
\hline
\end{tabular}


Table 2. Summary of intraoperative and final findings

\begin{tabular}{cccccccc}
\hline & & Frozen & CD20 & MIB-1 & Frozen HE Permanent & Final \\
Case & Age/Sex & HE & (R-IHC) & $($ R-IHC) & + +R-IHC & MIB-1 & Dx. \\
\hline 34 & $68 / F$ & CNSL & diffuse & $90 \%$ & CNSL & $100 \%$ & CNSL \\
$35^{*}$ & $86 / F$ & CNSL & diffuse & $90 \%$ & CNSL & $90 \%$ & CNSL \\
$36^{*}$ & $49 / F$ & CNSL & diffuse & $50 \%$ & CNSL & $60 \%$ & CNSL \\
$37^{*}$ & $62 / F$ & HGG or & diffuse & $90 \%$ & CNSL & $90 \%$ & CNSL \\
\hline
\end{tabular}

*: recut section from reserved frozen blocks 
Table 3. Representative rapid-IHC methods

\begin{tabular}{ccccccc}
\hline Author & Year & Method & $\begin{array}{c}\text { Ki-67 Ab } \\
\text { clone }\end{array}$ & Dilution & Total time(min) Reference \\
\hline Ichihara T, et al. & 1989 & Microwave & N.D. & N.D. & 13 & {$[7]$} \\
Richiter T, et al. & 1999 & EPOS & N.D. & N.D. & 12 & {$[8]$} \\
Kammerer U, et. al. 2001 & Modified EnVision & KISS & $1: 10$ & 12 & {$[9]$} \\
Haapasalo J, et al. & 2005 & Ultrarapid-ki67 kit & N.D. & N.D. & 14 & {$[10]$} \\
Monig SP, et. al. & 2006 & En Vision, Histofine & N.D. & N.D. & $10 ~ 13$ & {$[12]$} \\
Hatta H, et. al. & 2006 & $\begin{array}{c}\text { Intermittent } \\
\text { microwave }\end{array}$ & N.D. & N.D. & 15 & {$[13]$} \\
Hatta H, et al. & 2010 & Ultrasound & MIB-1 & 1:40 & 10 & {$[14]$} \\
Uzuka T, et al. & 2011 & Vectastain kit & MIB-1 & $1: 200$ & 70 & {$[11]$} \\
Toda Y, et al & 2011 & AC field & N.D. & N.D. & 21 & {$[15]$} \\
Tanino M, et al. & 2014 & AC field & MIB-1 & 1:100 & 16 & This study \\
\hline
\end{tabular}

N.D.; not determined 
Fig. 1
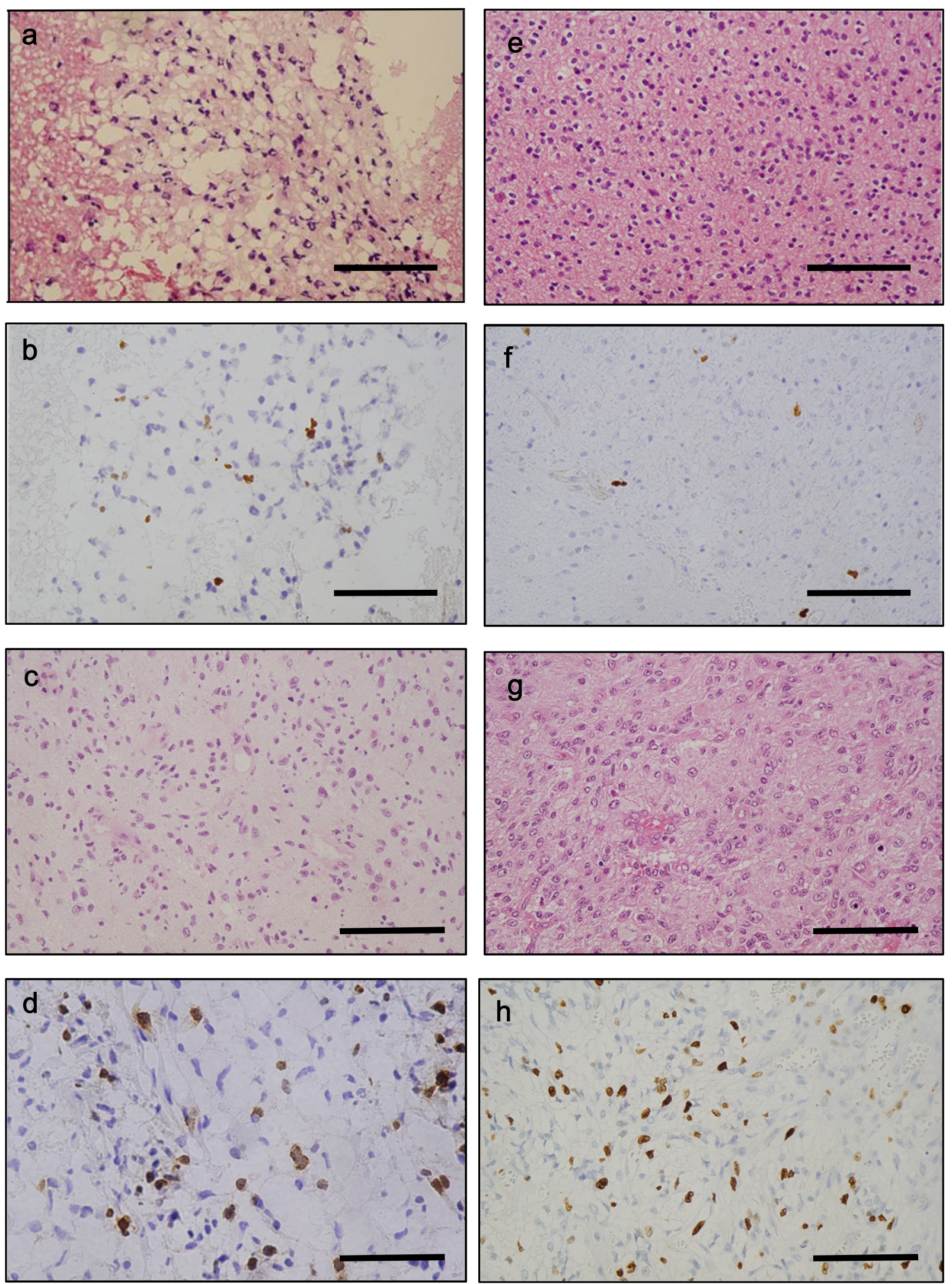
Fig. 2
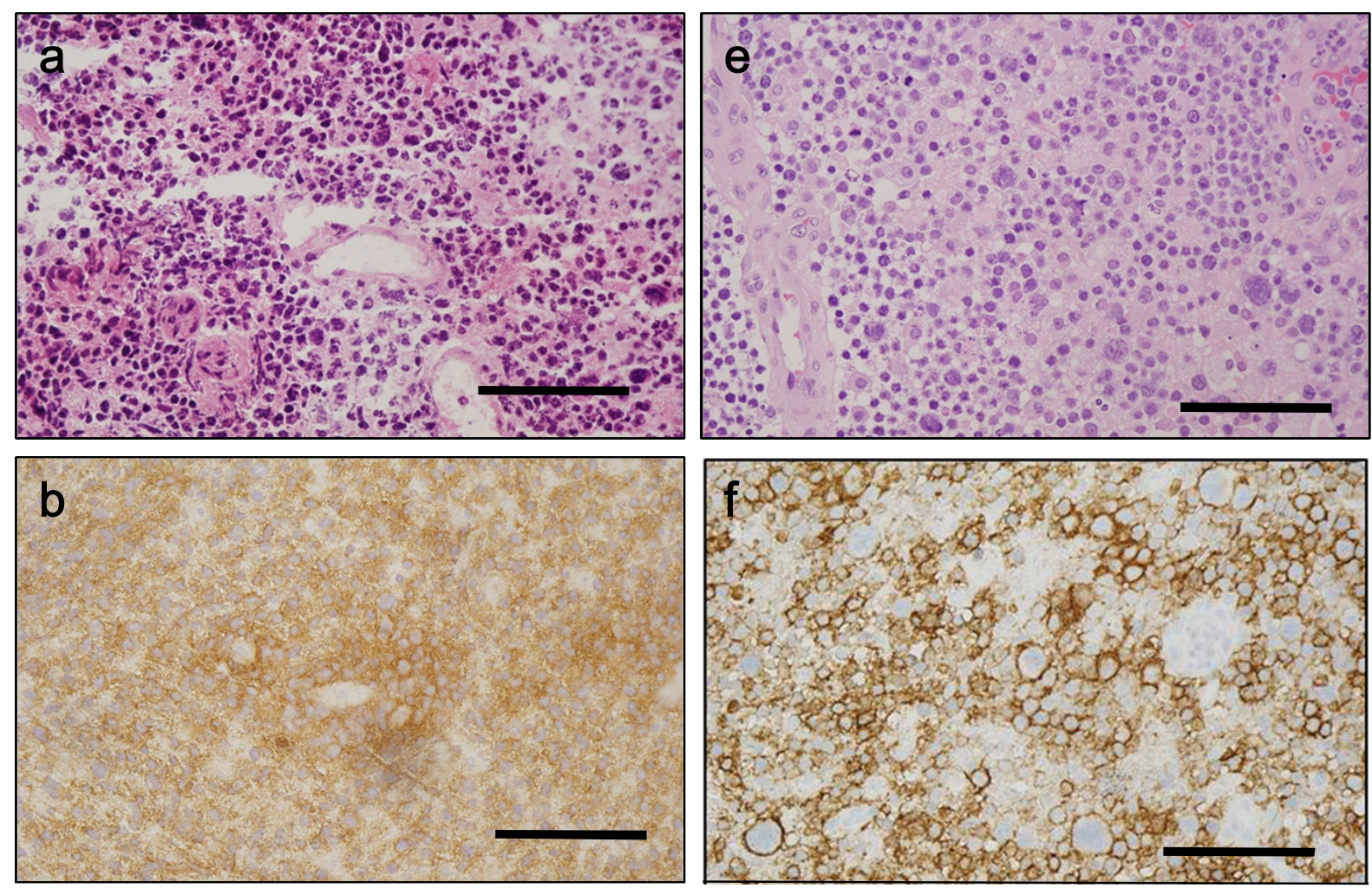

\section{c}
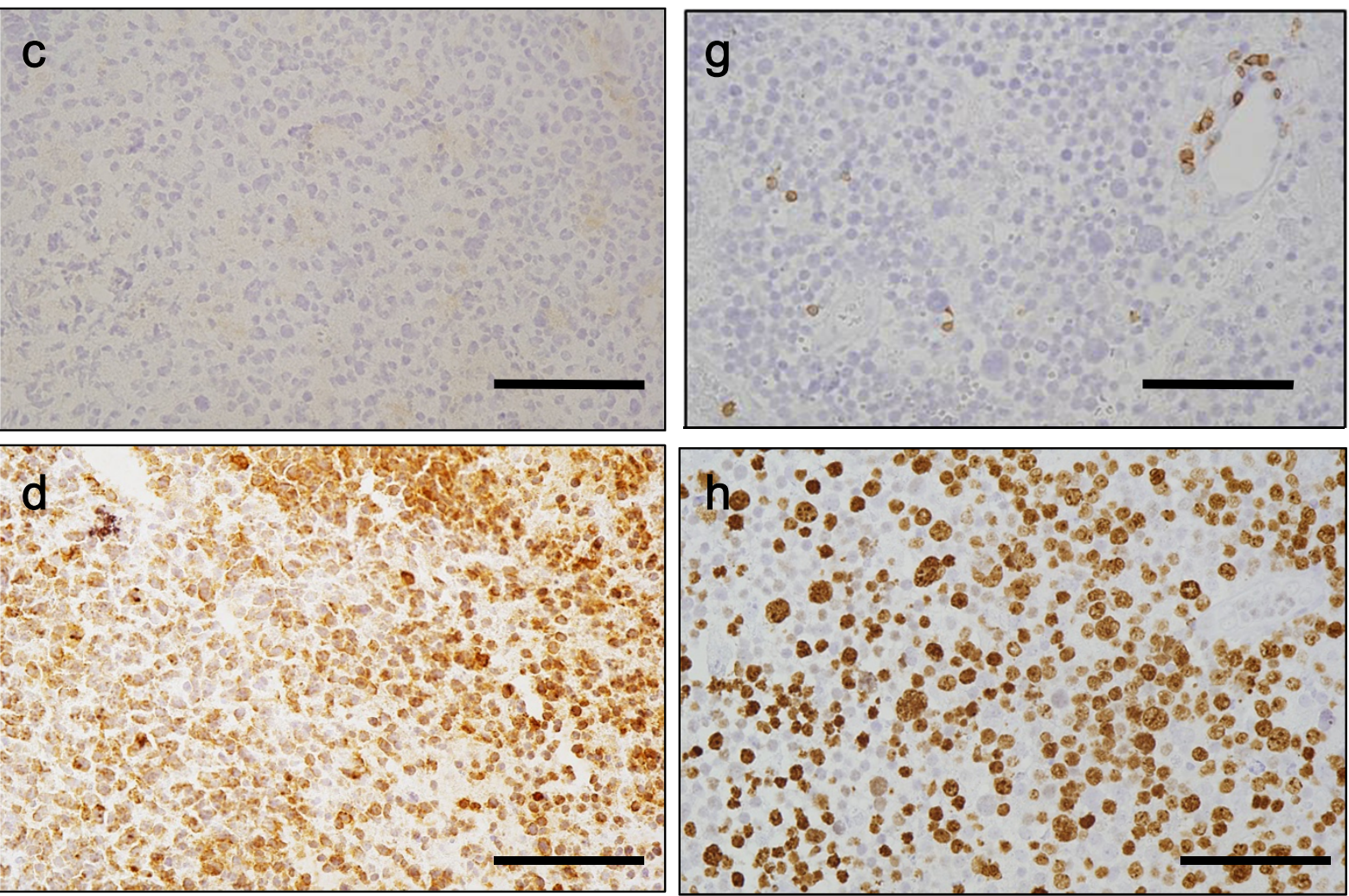
Fig. 3

a

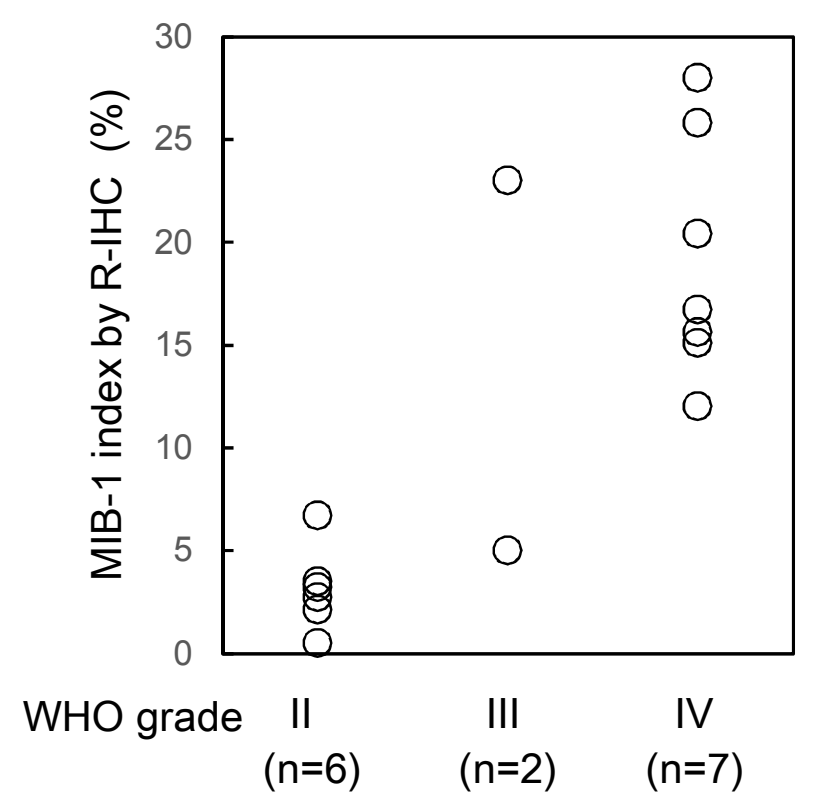

C

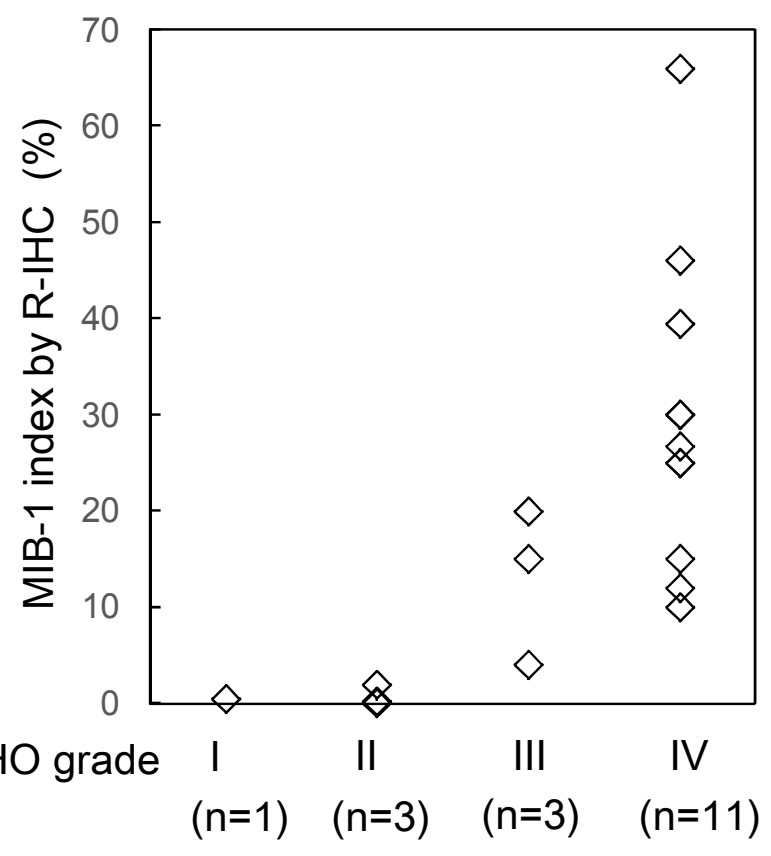

b
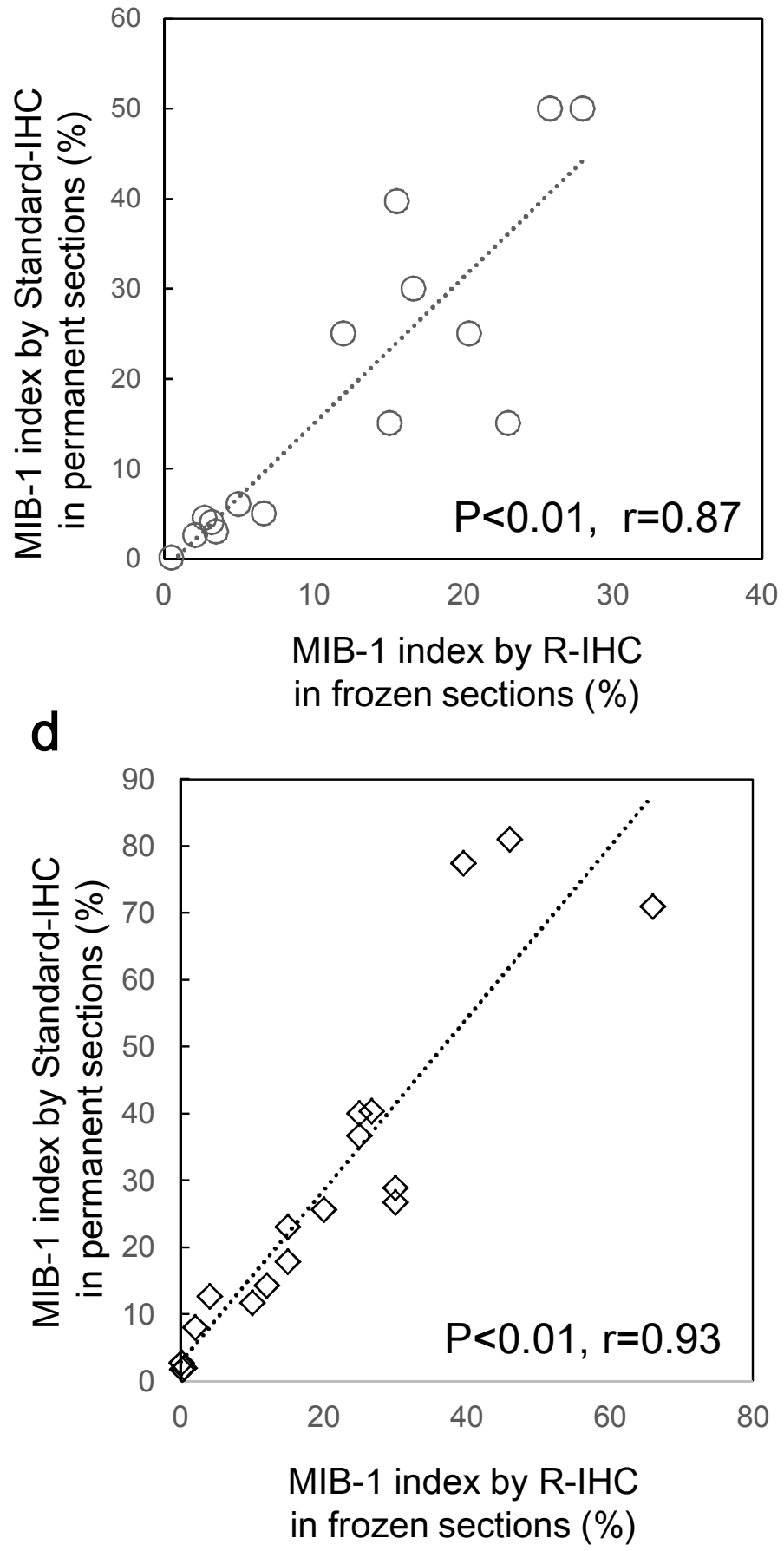